Ergod. Th. \& Dynam. Sys. (1987), 7, 463-479

Printed in Great Britain

\title{
A bound for the fixed point index of area-preserving homeomorphisms of two-manifolds
}

\author{
STEPHAN PELIKAN AND EDWARD E. SLAMINKA \\ Department of Mathematics, University of Cincinatti, Cincinnati, Ohio 45221, USA; \\ Department of Mathematics, Auburn University, Auburn, Alabama, USA
}

(Received 23 May 1985 and revised 14 March 1986)

\begin{abstract}
The study of area preserving maps of manifolds has an extensive history in the theory of dynamical systems. One interest has been in the behaviour of such maps near an isolated fixed point. In 1974 Carl Simon proved the existence of an upper bound for the index of an isolated fixed point for $C^{k}$ area preserving diffeomorphisms of a surface. We extend his result to homeomorphisms of an orientable two manifold. The proof utilizes the notion of free modification, developed by Morton Brown, and enlarges the scope of the problem to the consideration of 'nice' measures, i.e. uniformly equivalent to Lebesgue measure on compact sets. By suitably modifying the homeomorphism and the measure, we obtain the following theorem.
\end{abstract}

THEOREM. Let $h: M^{2} \rightarrow M^{2}$ be an orientation preserving homeomorphism of a smooth orientable two manifold which preserves area. If $p$ is an isolated fixed point of $h$, then the index of $p$ is $\leq+1$.

\section{0 . Introduction}

What restrictions does the hypothesis that a homeomorphism be area-preserving place on the dynamics of the map? In this paper we present a result about the possible dynamics of such a map in the neighbourhood of an isolated fixed point: the index of the point must be less than 2 .

This result may be viewed as a generalization of Simon's theorem [6], which is concerned with $C^{k}$ diffeomorphisms. Our method of proof is necessarily completely different from his, and appears to be applicable in a wide variety of situations. The method involves a procedure by which an area preserving homeomorphism is modified to produce a new homeomorphism which has the same fixed points, which preserves an equivalent measure, and which has a special canonical form. This procedure has its roots in the Brouwer Translation Arc Lemma, and employs the idea of a free modification of a homeomorphism developed by Brown [3], [4], and by Schmitt [5]. It should be noted that, although we are primarily concerned with homeomorphisms, the techniques we use work equally well in the setting of $C^{k}$ diffeomorphisms. 
Some of the applications of the results presented here derive from the fact that our theorem is a generalization of Simon's. In [6] Simon gives a number of applications of his theorem to mechanical (Hamiltonian) systems. In these applications a diffeomorphism of a surface is obtained from the flow in such a way that fixed points correspond to time periodic motions of the system. Simon then applies his result to conclude that the motions are nondegenerate, deducing, on topological grounds, the existence of other, independent, periodic motions.

As a result of theorem I below, his conclusions are true under the less restrictive assumption that the Hamiltonian governing the system is piecewise differentiable in such a way that a continuous, but not necessarily smooth flow is obtained. Piecewise smooth Hamiltonian systems, which are frequently easier to investigate numerically than their smooth analogues, have been receiving considerable attention in the physical literature.

In addition to their physical significance, area-preserving homeomorphisms have an intrinsic interest of their own. As an illustration of the usefulness of our theorem $I$ in answering global versions of the question with which this section began, we mention a corollary to theorem I due to $\mathrm{K}$. Boucher [1]:

COROllaRy. Let $h: S^{1} \rightarrow S^{1}$ be a homeomorphism which has at least $n$ attracting (and therefore at least $n$ repelling) fixed points. Then any extension of $h$ to an orientation preserving, area-preserving homeomorphism of the disc $D$ has at least Greatest integer $(n / 2+1)$ fixed points in the interior of $D$.

S. Pelikan was partially supported by the National Science Foundation. E. E. Slaminka was partially supported as a Charles P. Taft Postdoctoral Fellow, University of Cincinnati.

\section{Definitions and notation}

Let $\mathbb{R}^{2}$ denote the 2-plane with the standard norm \|\| .

A disc $D \subset \mathbb{R}^{2}$ is a homeomorph of the unit ball $\left\{x \in \mathbb{R}^{2} \mid\|x\| \leq 1\right\}$.

For $X \subset \mathbb{B}^{2}$ let int $(X)$, bd $(X), \bar{X}, X^{c}$ denote the interior, boundary, closure and complement of $X$ respectively.

If $h$ is a homeomorphism of $\mathbb{R}^{2}$, let Fix $(h)$ denote the fixed point set of $h$.

By a nice measure $\mu$ on a 2 -manifold we mean a measure which is absolutely continuous with respect to Lebesgue measure, zero on points and with Radon Nikodym derivative $d \mu / d x$ bounded above and below from 0 by constants on compact sets. For our purposes this last condition is equivalent to the existence of positive constants $K_{1}, K_{2}$ such that $K_{1} m(A) \leq \mu(A) \leq K_{2} m(A)$ where $A$ is a Lebesgue measurable set contained in some compact set and $m$ is Lebesgue measure.

A homeomorphism $h$ of $\mathbb{R}^{2}$ is said to preserve the measure $\mu$ if $\mu(A)=\mu(h(A))$ for all measurable sets $A$.

Definition. Given a simple closed curve $C$ in $\mathbb{R}^{2}$ and a homeomorphism $h: \mathbb{R}^{2} \rightarrow \mathbb{R}^{2}$ with $C \cap$ Fix $(h)=\varnothing$, the index of $C$ with respect to $h$, denoted ind $(h, C)$, is the 
degree (i.e. winding number) of the mapping $H: C \rightarrow S^{1}$ where

$$
H(x)=\frac{h(x)-x}{\|h(x)-x\|} .
$$

We shall need the following two basic properties of the index:

(i) Let $h$ and $g$ be two homeomorphisms such that Fix $(g)=$ Fix $(h)$ and let $C$ be a simple closed curve such that $C \cap \operatorname{Fix}(h)=\varnothing$. If there exists an isotopy $\Phi_{t}$ between $h$ and $g$ such that Fix $(h)=$ Fix $\left(\Phi_{t}\right)$ then ind $(h, C)=$ ind $(g, C)$.

(ii) If $C$ is invariant under $h$ then ind $(h, C)=1$.

Definition. If $p$ is an isolated fixed point for $h$, then let the index of $p$, denoted ind $(h, p)$, be ind $(h, C)$ for a simple closed curve $C$ which bounds a disc $D$ containing $p$, where $D \cap$ Fix $(h)=p$. It follows easily that the index of a fixed point is well-defined.

By the Brouwer fixed point theorem one proves that if ind $(h, C) \neq 0$ for some simple closed curve $C$, then the disc $D$ bounded by $C$ contains a fixed point for $h$.

The relation between index of a curve and index of a point is as follows:

LEMMA. Let $h$ be a homeomorphism of the plane with isolated fixed point set. If $C$ is a simple closed curve with $C \cap \operatorname{Fix}(h)=\varnothing$, then $\sum$ ind $(h, p)=$ ind $(h, C)$ where the sum is taken over those fixed points contained in the disc bounded by $C$.

\section{The main theorem}

This section presents a statement of the main theorem, a reduction of this theorem, and introduces the notion of a free modification of a homeomorphism. Much of this section depends on the following form of the Brouwer lemma, which is due to Brown [2]:

BrouWER LEMMA. Let $h: \mathbb{R}^{2} \rightarrow \mathbb{R}^{2}$ be an orientation preserving homeomorphism of the plane, and let $D$ be a disc with $h(D) \cap D=\varnothing$. If $h^{n}(D) \cap D \neq \varnothing$ for some $n \neq 0$, then there exists a simple closed curve $C$ with $C \cap$ Fix $(h) \neq \varnothing$ such that the index of $C$ is 1 .

THEOREM I. Let $h: M^{2} \rightarrow M^{2}$ be an orientation preserving homeomorphism of a smooth orientable 2 manifold $M^{2}$ which preserves area. If $p$ is an isolated fixed point of $h$, then the index of $h$ at $p$ is less than or equal to 1.

Proof. Assume that there exists an isolated fixed point $p$ of $h$ with ind $(h, p)=n>1$. Let Fix $(h)=F \cup\{p\}$. Since Fix $(h)$ is closed and $p$ is isolated, $F$ is a closed set. Consider the connected component $X$ of $M^{2}-F$ containing the point $p$. Since $M^{2}$ is orientable it follows that the universal cover $\tilde{X}$ of $X$ is either $\mathbb{R}^{2}$ or $S^{2}$, and that $h$ lifts to a homeomorphism $\tilde{h}: \tilde{X} \rightarrow \tilde{X}$ which fixes only the fiber $\pi^{-1}(p)$, where $\pi: \tilde{X} \rightarrow X$ is the covering projection. Since $\pi$ is a local homeomorphism, $\pi^{-1}(p)$ consists of a countable collection of isolated points $p_{1}, p_{2}, \ldots$, and ind $\left(\tilde{h}, p_{i}\right)=n$ for all $i$ such that $p_{i}$ is fixed under $\tilde{h}$.

The case where the universal cover is $S^{2}$ will be handled separately. Since $X$ is orientable $X \approx S^{2}$. Hence the only fixed point for $h$ is $p$ with index 2 . However, by 
using the Brouwer Translation Arc Lemma on $S^{2}-p \approx$ int $(D)$, there exists at least one other fixed point, a contradiction.

We will now define a measure $\mu$ on $\mathbb{R}^{2}$ which is invariant under $\tilde{h}$. If $A \subset \mathbb{R}^{2}$ is such that $\pi$ is one to one on both $A$ and $\tilde{h}(A)$, set $\mu(A)=$ area $(\pi(A))$. Then $\mu$ is invariant because if $A$ has the property that $\pi$ is one to one on $A$ and $\tilde{h}(A)$, then

$$
\mu(\tilde{h}(\boldsymbol{A}))=\operatorname{area}(\pi \tilde{h}(A))=\operatorname{area}(h \pi(A))=\operatorname{area}(\pi(A))=\mu(A) .
$$

The fact that $\mu$ is nice follows from the fact that $\pi$ can be chosen to be a smooth map of $\mathbb{R}^{2}$ to $X$. Thus without loss of generality assume that there exists an orientation preserving homeomorphism $h: \mathbb{R}^{2} \rightarrow \mathbb{R}^{2}$ which preserves a nice measure $\mu$ and such that:

(i) $\operatorname{Fix}(h)=\left\{p_{1}, p_{2}, \ldots\right\}$ is isolated; and,

(ii) ind $\left(h, p_{i}\right)=n>1$ for all $i$.

The proof of Theorem I then reduces to the following theorem.

THEOREM II. Let $h: \mathbb{R}^{2} \rightarrow \mathbb{R}^{2}$ be an orientation preserving homeomorphism of the plane which preserves a nice measure. If $\mathrm{Fix}(h)=\left\{p_{1}, p_{2}, \ldots\right\}$ where each $p_{i}$ is isolated and ind $\left(h, p_{i}\right)=n$ for all $i$, then $n \leq 1$.

We prove this theorem by contradiction, assuming that $n>1$. It will be necessary to consider free modifications of the given homeomorphism $h$. Each free modification $h f$ of $h$ has the same fixed point set as $h$ and any simple closed curve $C$ with $C \cap$ Fix $(h)=\varnothing$ has ind $(h, C)=$ ind $(h f, C)$.

We will show that under suitable conditions one can modify a homeomorphism to one which, though it does not preserve the original measure, nevertheless, preserves another nice measure. The following discussion concerning free modifications and reduction to canonical form is due to Brown [2], [3] and Schmitt [5].

Definition ([2]). Two homeomorphisms $h$ and $g$ of $\mathbb{R}^{2}$ are said to be strongly equivalent provided there exists a disc $D \subset \mathbb{R}^{2}$ such that $h(D) \cap D=\varnothing$ and $g^{-1} h$ is supported on $D$ (i.e. $g^{-1} h(x)=x$ for all $x \in D$ ). We say that $h$ is freely equivalent to $g$ if there exists a finite sequence of homeomorphisms $h_{0}, \ldots, h_{n}$ such that $h=h_{0}, g=h_{n}$ and $h_{i}$ is strongly equivalent to $h_{i+1}$. One observes that if $h$ is freely equivalent to $g$, then Fix $(h)=$ Fix $(g)$.

Given a homeomorphism $h$ of $\mathbb{R}^{2}$ we will construct a freely equivalent homeomorphism by a finite sequence of free modifications.

Definition. A homeomorphism $f$ of $\mathbb{R}^{2}$ is a free modification of $h$ provided that there exists a disc $D$ in $\mathbb{R}^{2}$ such that $h(D) \cap D=\varnothing$ and $f$ is supported on int $(D)$.

By Alexander's Isotopy Theorem $f$ is isotopic to the identity by an isotopy $\Phi_{t}$ such that $\Phi_{t}(x)=x$ for $x \notin$ int $(D)$. We see that ind $(h, C)=$ ind $(h f, C)$ for any simple closed curve $C$ with $C \cap$ Fix $(h)=\varnothing$.

When a homeomorphism is modified, however, one does not know a priori that there exists a nice measure which this new homeomorphism preserves. The construction of such a measure is possible under the hypotheses of theorem II. 
Proposition. Let $h$ be an orientation preserving homeomorphism of $\mathbb{R}^{2}$ preserving a nice measure $\mu$ and let $\mathrm{Fix}(h)$ be isolated with ind $(h, p)=n>1$ for each $p \in \operatorname{Fix}(h)$. Suppose that $h$ is strongly equivalent to $h f$ by a free modification $f$ on a disc $D$, and that $f$ is a $C^{2}$ diffeomorphism. Then there exists a nice measure $\nu$ preserved by $h f$.

Proof. For a measurable set $A \subset \mathbb{R}^{2}$ define $A_{j}=h^{-j}(D) \cap A$ for $j=0,1,2, \ldots$ and set $A_{c}=A-\bigcup_{j} A_{j}$. Then $A=A_{c} \cup \bigcup_{j} A_{j}$. We claim that we have expressed $A$ as the disjoint union of subsets. If this were not the case, then by the Brouwer Lemma there would exist a simple closed curve $C$ with index equal to 1 . However, the index of each simple closed curve in $\mathbb{R}^{2}$ is either 0 or a positive integral multiple of $n$. Define the measure $\nu$ as follows:

$$
\nu(A)=\mu\left(A_{c}\right)+\sum_{j=0}^{\infty} \mu\left((h f)^{j+1}\left(A_{j}\right)\right) .
$$

In order to show that $\nu$ is a nice measure, it suffices to obtain bounds on $\nu\left(A_{j}\right)$ in terms of the Lebesgue measure $m\left(A_{j}\right)$ which are uniform. Note that

$$
\mu\left((h f)^{j+1}\left(A_{j}\right)\right)=\mu\left(h f h^{j}\left(A_{j}\right)\right)=\mu\left(f h^{j}\left(A_{j}\right)\right),
$$

since $h$ preserves $\mu$.

Using the change of variables formula for integrals,

$$
\begin{aligned}
\mu\left(f h^{j}\left(A_{j}\right)\right) & =\int \chi_{f h^{j}\left(A_{j}\right)}(x) \frac{d \mu}{d x}(x) d x=\int \chi_{h^{j}\left(A_{j}\right)}\left(f^{-1} x\right) \frac{d \mu}{d x}(x) d x \\
& =\int \chi_{h^{j}\left(A_{j}\right)}(u) \frac{d \mu}{d x}(f(u))|\operatorname{det} D f(u)| d u .
\end{aligned}
$$

Using the fact that $d \mu / d x$ and $\mid \operatorname{det} D f(u)) \mid$ are bounded from zero and above by some constant on the compact set $h(D)$ shows that

$$
K_{1} \text { Lebesgue }\left(A_{j}\right) \leq \nu\left(A_{j}\right) \leq K_{2} \text { Lebesgue }\left(A_{j}\right)
$$

for constants $K_{1}$ and $K_{2}$ independent of $j$ and $A_{j}$, and hence that the measure $\nu$ is also a nice measure.

The above condition that $f$ be $C^{2}$ is not necessary. One could require only that $f$ be $L$-bi-Lipschitzian, that is

$$
(1 / L)|x-y| \leq|f(x)-f(y)| \leq L|x-y|
$$

for some constant $L \geq 1$. Then one can also obtain the inequality $(*)$.

Remark. To ensure that the measure $\nu$ is non-atomic it is essential that a bound be placed on the Jacobian as the following example shows:

Let $h(x, y)=(x / 2,2 y)$ and $D$ be the disc $\left\{(x, y)|| x\left|\leq \frac{1}{2},\right| y-\frac{3}{2} \mid \leq \frac{1}{2}\right\}$. Let $f: D \rightarrow D$ be the free modification defined such that the vertical lines $x= \pm 2^{-n}, n=1,2, \ldots$ are mapped as shown in figure 1 . Now extend $f$ so that $f$ restricted to the boundary of $D$ is the identity. 

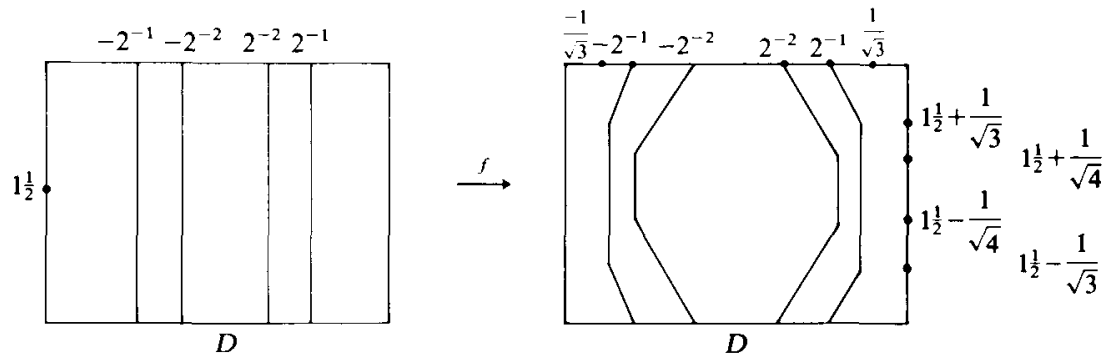

$$
\left( \pm 2^{-n}, 1 \frac{1}{2}\right) \mapsto\left(\frac{ \pm 1}{\sqrt{n+2}}, 1^{\frac{1}{2}}\right)
$$

FIGURE 1

Let $E=\left\{(x, y)|| x\left|\leq \frac{1}{2}, \quad\right| y \mid \leq \frac{1}{2}\right\}$. Then $F_{n}=h^{n}\left(h^{-n}(D) \cap E\right)=\left\{(x, y)|| x \mid \leq 2^{-n}\right.$, $\left.\left|y-\frac{3}{2}\right| \leq \frac{1}{2}\right\}$. See figure 2. After modifying $h$ by $f$ we have that

$$
\nu(E) \geq \nu\left(\cup F_{n}\right)=\sum \nu\left(F_{n}\right)=\sum m\left(f F_{n}\right)>\sum 1 / n=\infty .
$$

Thus any neighbourhood of the origin will have infinite measure, and hence the measure $\nu$ will be atomic.

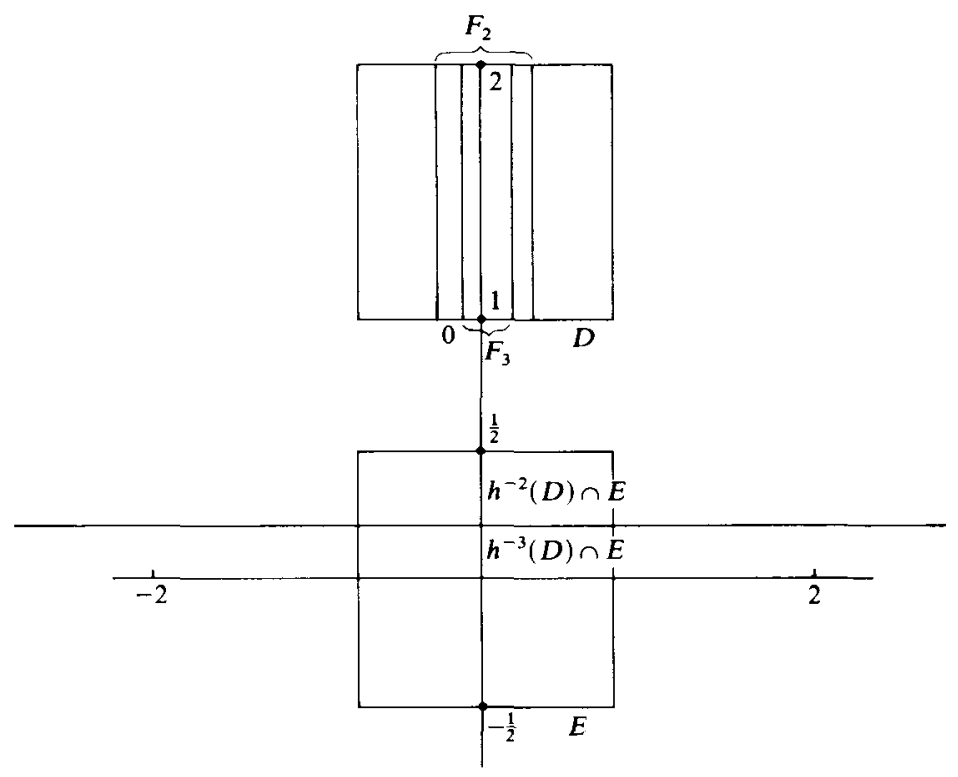

FIGURE 2

In reducing $h$ to canonical form free modifications will be made on maps which preserve a nice measure. Each modification will be constructed using only maps which are $C^{2}$ diffeomorphisms with Jacobian bounded above and below from 0 (or $L$-bi-Lipschitzian maps). Thus, each of these modifications will result in a new homeomorphism which preserves a new (but nice) measure. 


\section{Canonical form and the proof of Theorem II}

The index of a curve $C$ with respect to an orientation preserving homeomorphism $h$ can be calculated by considering the rotation of the vector $h(x)-x$ as $x$ passes through two consecutive points on $C$ whose images also lie on $C$. If the rotation is approximately $-\pi$ or $\pi$, one counts a contribution of +1 or -1 to the index. Summing over such pairs of points, and adding 1 for the total rotation of $C$ yields the index of $C$. Some pairs of points which contribute 0 to the index are illustrated in figure 3. An arc on $C$ with endpoints $a$ and $b$ having the property that $h(a)$ and $h(b)$ are
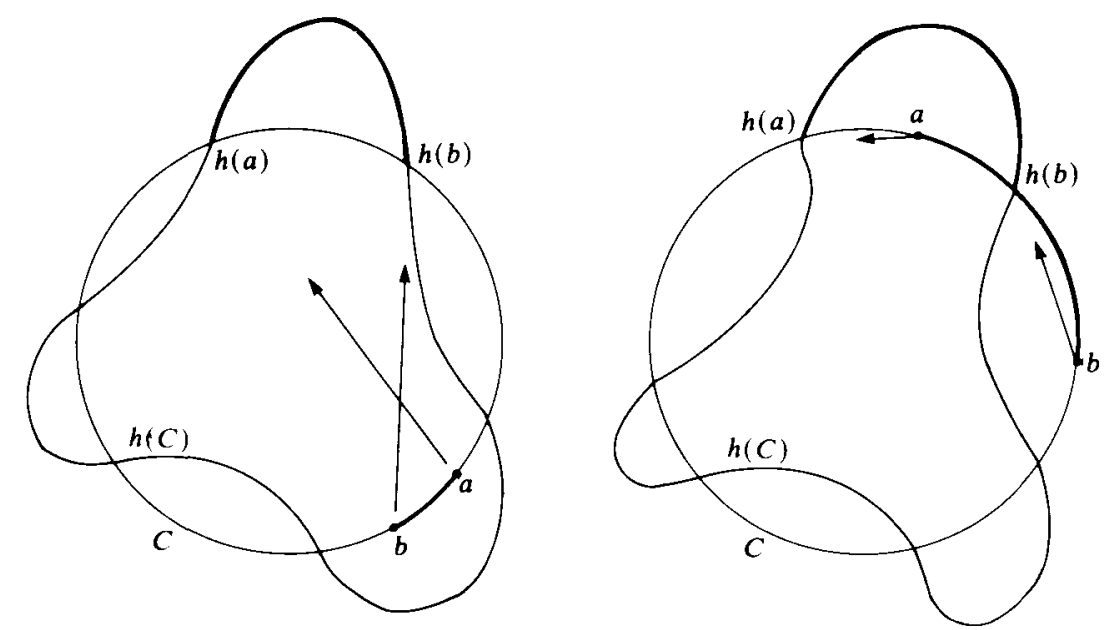

FIGURE 3

also on $C$ and that the arc on $C$ from $h(a)$ to $h(b)$ contains the arc from $a$ to $b$ (see figure $4 a$ ) is called an elliptic arc. Elliptic arcs contribute +1 to the index of $C$. If the arc from $h(a)$ to $h(b)$ is contained in the arc from $a$ to $b$, the arc is called hyperbolic (see figure 4b). Hyperbolic arcs contribute -1 to the index of $C$. We can

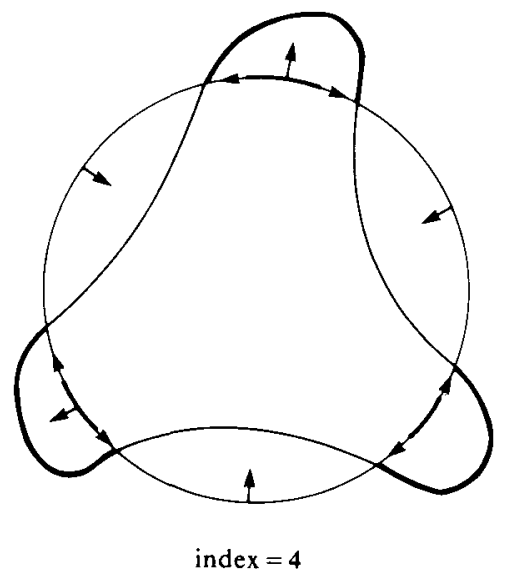

(a)

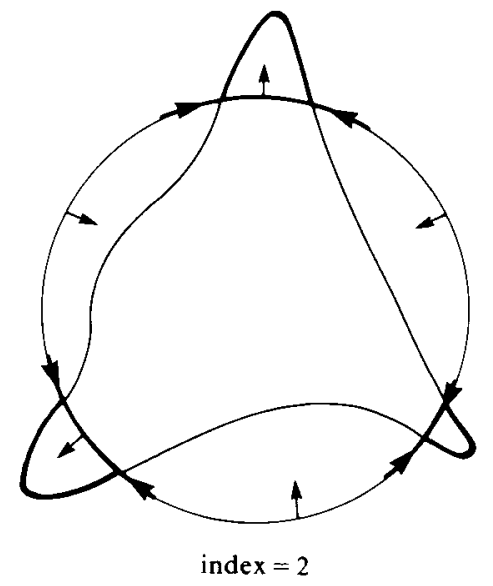

(b)

Figure 4 
then compute that ind $(h, C)=1+e-h$, where $e$ is the number of elliptic arcs on $C$, and $h$ the number of hyperbolic arcs.

Definition. A homeomorphism $f$ freely equivalent to $h$ is a canonical form of $h$ on the curve $C$ if the number of intersections of $C$ with $f(C)$ is exactly $|2(n-1)|$, where $n=$ ind $(h, C)$.

Thus, if ind $(h, C)=4$, a canonical form of $h$ on $C$ would have exactly 3 elliptic arcs.

In $\S 4$ it will be shown that, under the hypotheses of theorem II, every homeomorphism has a canonical form which preserves a nice measure. Using this fact. we first prove theorem II.

Proof of Theorem II. Assume that $n \geq 2$, and let $C$ be a simple closed curve disjoint from Fix $(h)$ and with the property that if $D$ is the disc bounded by $C$, then $D \cap \operatorname{Fix}(h)=\left\{p_{1}\right\}$. Without loss of generality, we may assume that $h$ is in canonical form on $C$. Since ind $(h, C)=n>1, h(C)$ consists of $n-1$ elliptic arcs $\alpha_{i}$ with endpoints $a_{i}, b_{i}$ such that the $\operatorname{arc} a_{i} b_{i} \subset C \cap h(D)$. Let $E_{i}$ be the disc bounded by $\alpha_{i} \cup a_{i} b_{i}$.

Let $x \in$ int $\left(\alpha_{1}\right)$. We will construct an arc $J$ lying in $D$ such that $J$ is a subset of an invariant line through $x$, and $J$ intersects $C$ at two points, $h^{-1}(x)$ and $y$. Then we will consider the simple closed curve $J \cup K$ where $K \subset C$ is an arc with endpoints $h^{-1}(x)$ and $y$. A simple computation will show that $1 \leq$ ind $(h, J \cup K) \leq n-1$, which will be a contradiction and complete the proof.

Let $x$ be chosen as above, and $F$ a disc in $E_{1}$ such that $x, h^{-1}(x) \in F$. Note that $h^{-1}(F) \subset D$. Since int $(F) \cap h^{-1}($ int $(F))=\varnothing$, by the Brouwer Lemma int $(F) \cap$ $h^{n}$ (int $\left.(F)\right)=\varnothing$ for all $n \neq 0$. However, $\mu(F)>0$ and $\mu(D)<\infty$ which implies that there exists an $N>0$ such that $h^{-N}(F) \cap C \neq \varnothing$. Choose the smallest such $N$ with this property. Let $l_{1}$ be an arc from $h^{-N}(x)$ to some point $y \in C$ such that int $\left(l_{1}\right) \subset$ int $\left(h^{-N}(F) \cap D\right)$. Let $l_{2}$ be an arc from $y$ to $h^{-(N+1)}(x)$ such that int $\left(l_{2}\right) \subset$ int $\left(h^{-N}(F)\right)$ and $l_{1} \cup l_{2}=y$. (See figure 5.) The arc $J^{\prime}=h\left(l_{1}\right) \cup \bigcup_{i=2}^{N} h^{i}\left(l_{1} \cup l_{2}\right)$ lies

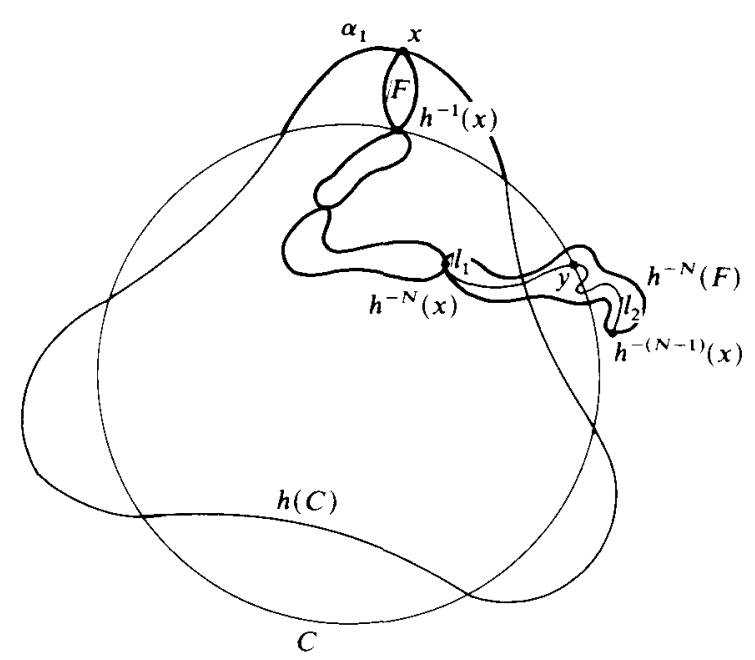

FIGURE 5 
in $h(D)$ and $J^{\prime} \cap h(C)=\{h(y), x\}$. Let $J=h^{-1}\left(J^{\prime}\right)$ and let $K_{1}, K_{2} \subset C$ be the arcs with endpoints $\left\{y, h^{-1}(x)\right\}$. Then $J \cup K_{i}$ is a simple closed curve for $i=1,2$. (See figure 6.)

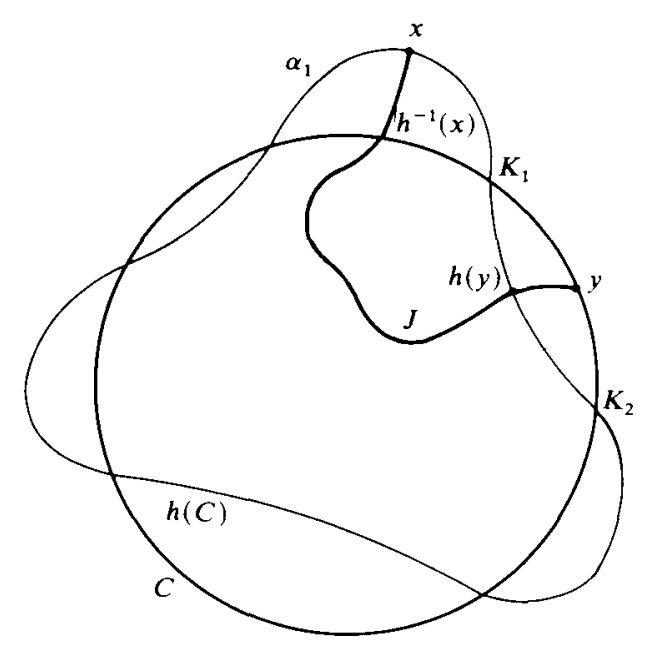

Figure 6

LEMMA. $1 \leq$ ind $\left(h, J \cup K_{i}\right) \leq n-1$ for $i=1,2$.

Proof. Either by direct computation, noting that the index is determined by the number of elliptic arcs which intersect $h\left(K_{i}\right)$, or by using free modifications to eliminate all but one intersection of $h(J)$ with $J$.

Remark. The above method works as well with $n$ hyperbolic arcs. The index of $h$ on $C$ is then $1-n$. The last lemma then would state that the index of $J \cup K_{i}$ is between 0 and $-n$. The arc $J$ must then pass through $C$ between $\alpha_{1}$ and either $\alpha_{2}$ or $\alpha_{n}$. We then have that the simple closed curve $J \cup K_{i}$ has index 0 which presents no contradiction. This is precisely the case for the smooth hyperbolic maps of the plane. As an example consider the smooth map $(x, y) \rightarrow(x / 2,2 y)$.

\section{Reduction to canonical form}

The following procedure will remove pairs of intersection points which contribute 0 to the total index. These types are indicated in figure 3 .

We first assume that $h(C)$ intersects $C$ transversely and only finitely often, and that the disc bounded by $C$ intersects its image in only one component. Let $D$ be the disc bounded by $C$, and consider the arcs $\alpha_{1}, \ldots, \alpha_{m}$ with $\bigcup \alpha_{i}=\overline{h(C) \cap D^{c}}$. Let $\alpha_{i}$ have endpoints $a_{i}, b_{i}$ and $a_{i} b_{i}$ be the arc on $C$ such that $a_{i} b_{i} \subset h(D) \cap C$. (See figure 7.) 


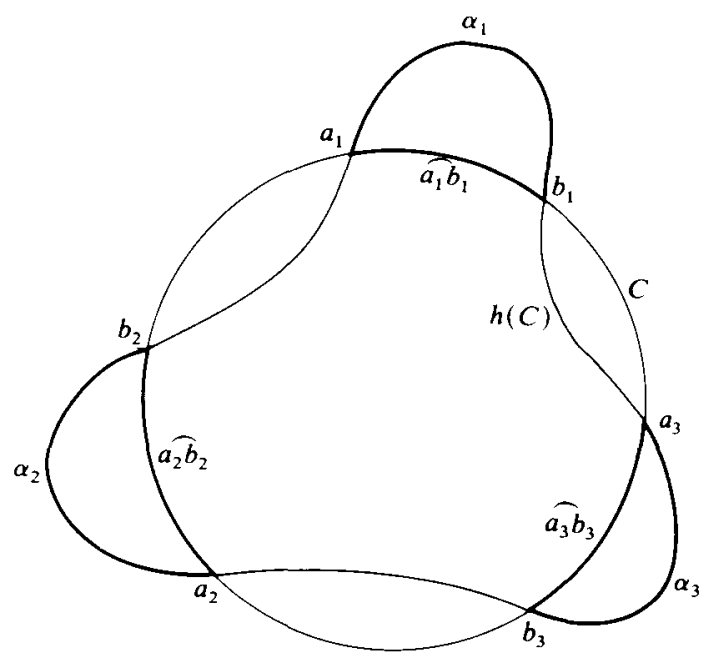

FIGURE 7

Case I. Assume that $h^{-1}\left(\alpha_{i}\right) \cap a_{i} b_{i}=\varnothing$. Since $a_{i} b_{i}-\left\{a_{i}, b_{i}\right\}$ lies entirely within int $(h(D))$ we have that $h^{-1}\left(a_{i} b_{i}-\left\{a_{i}, b_{i}\right\}\right)$ lies entirely within int $(D)$. Thus we can find a disc $D\left(\alpha_{i}\right)$ containing $\alpha_{i} \cup a_{i} b_{i}$ such that $\left.h^{-1}\left(D\left(\alpha_{i}\right)\right) \cap D\left(\alpha_{i}\right)\right)=\varnothing$. We then employ a free modification $f$ on $h^{-1}\left(D\left(\alpha_{i}\right)\right)$ such that $h f\left(h^{-1}\left(\alpha_{i}\right)\right) \subset$ int $(D)$. (See figure 8.) With this modification $h f(C)$ has two fewer points of intersection with $C$.

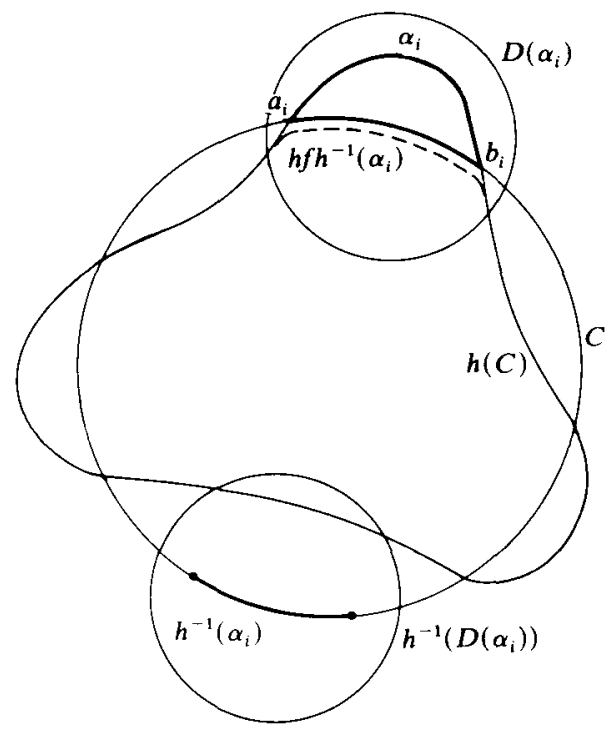

FIGURE 8

Case II. Assume that $h^{-1}\left(\alpha_{i}\right) \cap a_{i} b_{i} \neq \varnothing$ and precisely one of $a_{i}$ or $b_{i}$ lie in $h^{-1}\left(\alpha_{i}\right)$. Assume $b_{i}$ lies in $h^{-1}\left(\alpha_{i}\right)$. Since $h$ is orientation preserving $h^{-1}\left(a_{i}\right) \in a_{i} b_{i}$ (figure 9a). Let $d_{i} \in \alpha_{i}$ be a point near $b_{i}$ and $\tilde{\alpha}_{i} \subset \alpha_{i}$ the arc from $a_{i}$ to $d_{i}$. Pick a disc $D\left(\tilde{\alpha}_{i}\right)$ 


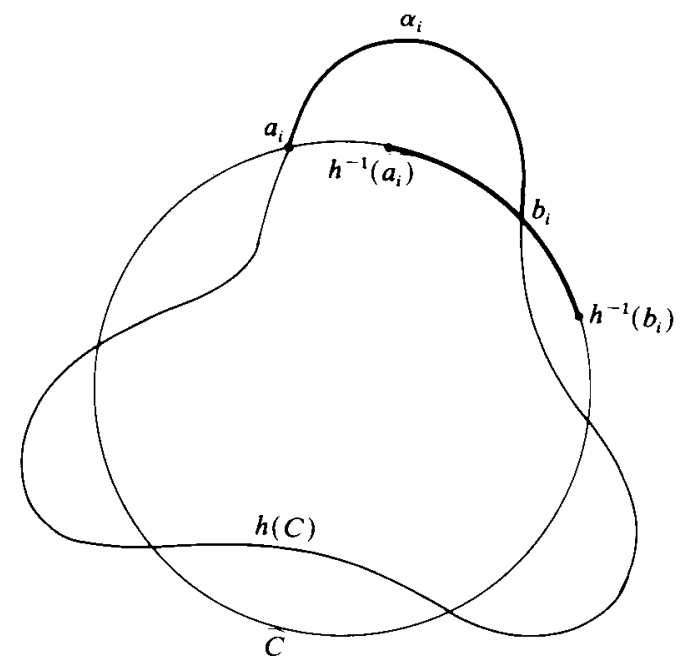

FIGURE 9(a).

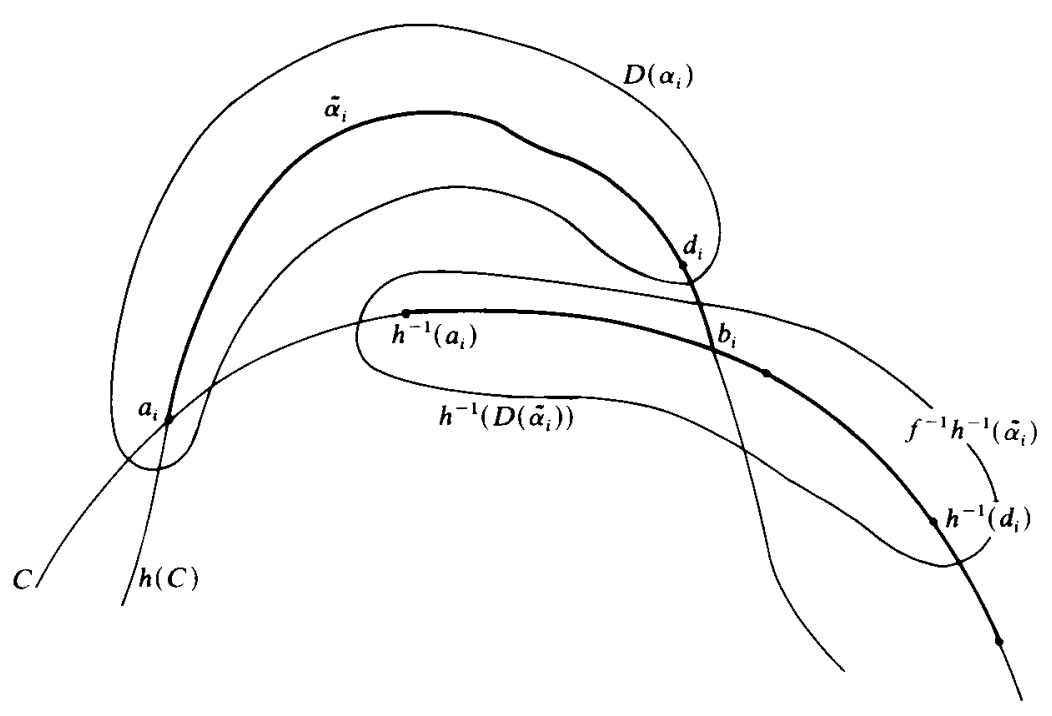

FigurE 9(b).

containing $\tilde{\alpha}_{i}$ such that $D\left(\tilde{\alpha}_{i}\right) \cap h^{-1}\left(D\left(\tilde{\alpha}_{i}\right)\right)=\varnothing$. Modify $h$ on $h^{-1}\left(D\left(\tilde{\alpha}_{i}\right)\right)$ by $f$ such that $f\left(h^{-1}\left(\tilde{\alpha}_{i}\right)\right) \cap \alpha_{i}=\varnothing$. (Figure 9b.) We now have that $(f h)^{-1}\left(\alpha_{i}\right) \cap a_{i} b_{i}=\varnothing$ and use Case I to eliminate two intersection points. Similarly if $h^{-1}\left(\alpha_{i}\right) \cap a_{i} b_{i} \neq \varnothing$ and both $a_{i}$ and $b_{i}$ lie in $h^{-1}\left(\alpha_{i}\right)$, but $h^{-1}\left(\alpha_{i}\right) \neq a_{i} b_{i}$, use the above method to eliminate two intersection points. (See figure 10.) 


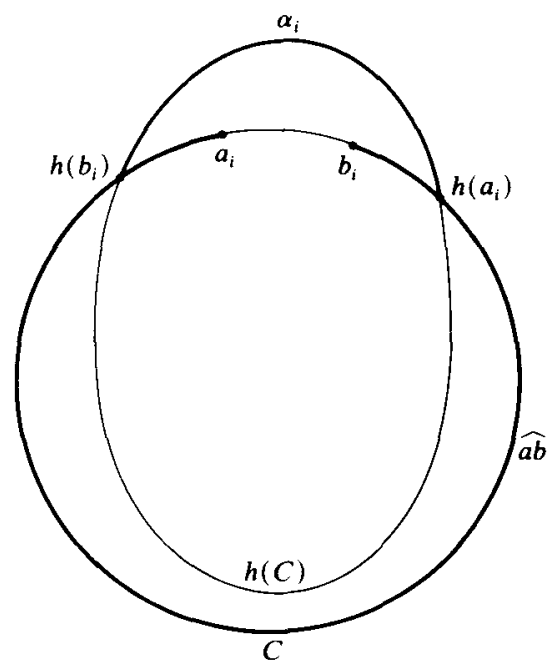

FIGURE 10

Case III. We are now left with arcs $\alpha_{i}$ such that either $h^{-1}\left(\alpha_{i}\right) \subset a_{i} b_{i}, a_{i} b_{i} \subset h^{-1}\left(\alpha_{i}\right)$ or $h(C) \cap C=\varnothing$. Since $h$ is orientation preserving and fixed point free on $C$, these containments are proper. Call those $\alpha_{i}$ such that $h^{-1}\left(\alpha_{i}\right) \subset a_{i} b_{i}$ elliptic arcs and call those $\alpha_{i}$ such that $a_{i} b_{i} \subset h^{-1}\left(\alpha_{i}\right)$ hyperbolic arcs.

Assume that an elliptic arc is adjacent to a hyperbolic arc along $C$. Let $a_{1}, b_{1}$, $a_{2}, b_{2}$ be the four successive intersections of $h(C)$ with $C$, where $a_{1}, b_{1}$ are the endpoints of the elliptic arc and $a_{2}, b_{2}$ are the endpoints of the hyperbolic arc. Consider the arc $\beta$ along $h(C)$ from $b_{1}$ to $a_{2}$. From the point of view of $h^{-1}$, this arc is in Case II, wherein we 'pull' $\beta$ outside of $D$. We now have an arc with endpoints $a_{1}, b_{2}$ which can be removed as in Case II. This procedure removes four intersection points. (See figure 11.)

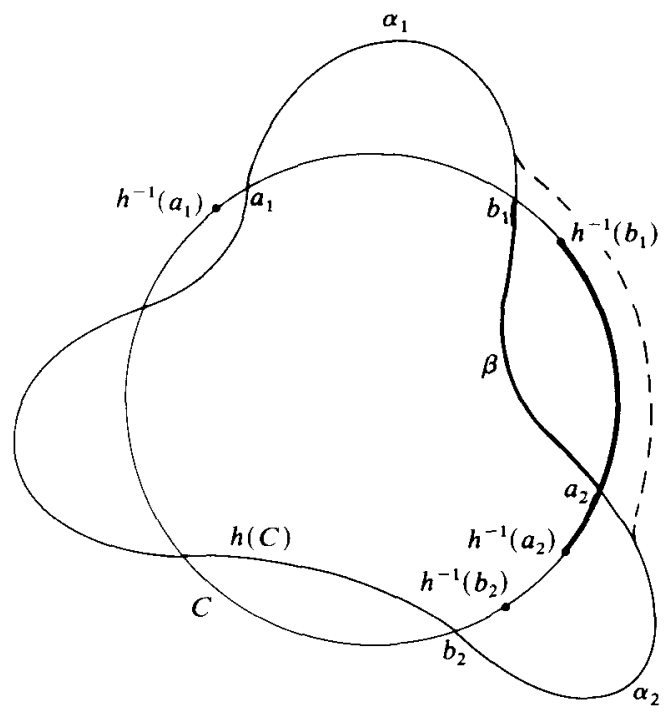

Figure 11 
We are left with a curve $C$, after a finite number of free modifications, which is either disjoint from its image, is composed of a finite number of elliptic arcs, or is composed of a finite number of hyperbolic arcs. One computes the index as $1,1+e$ or $1-h$, where $e$ is the number of elliptic arcs and $h$ is the number of hyperbolic arcs.

In the more general setting where $h(C) \cap C$ consists of nontransverse intersections we consider a Lebesgue number $\delta$ for $h$ restricted to $C$ such that if $I$ is any connected arc on $C$ with diameter less than $\delta$, then $h(I) \cap I=\varnothing$. Pick a finite collection of such arcs which cover $C$. For each such $I$ we will modify $h$ on a disc $D(I)$ containing $I$ in its interior which will replace the nontransverse intersection by a transverse one on $I$. (See figure 12.) The dotted line represents the image of an open arc containing $I$ in $D(I)$ under the free modification $f$. Since $C$ is covered by finitely many such intervals, we need perform this operation only finitely many times.

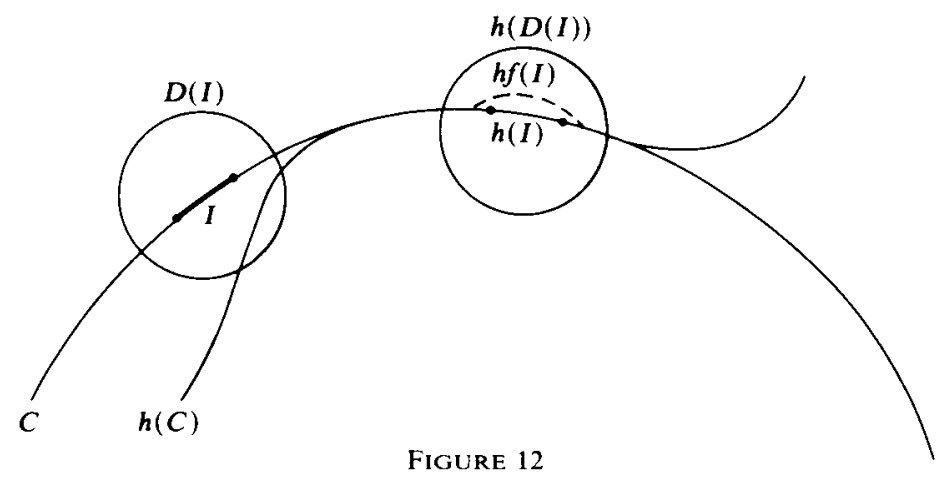

In case $h(C) \cap C$ consists of an infinite number of transverse intersections, cover $h(C) \cap C$ with a finite number of discs $D_{1}, D_{2}, \ldots, D_{k}$ having the properties that (1) $h^{-1}\left(D_{i}\right) \cap D_{i}=\varnothing$ for each $i$, and (2) $h(C) \cap D_{i}$ is connected for each $i$. Connect the endpoints of $C \cap h^{-1}\left(D_{i}\right)$ by a smooth arc $\alpha$ which lies in $h^{-1}\left(D_{i}\right)$ and which intersects $h^{-1}\left(C \cap D_{i}\right)$ a finite number of times. (This can be done by an arc having at most one point of intersection.) Modify $h$ on $h^{-1}\left(D_{i}\right)$ by a map which takes $C \cap h^{-1}\left(D_{i}\right)$ into the arc $\alpha$. After a finite number of such modifications we obtain a map freely equivalent to $h$ under which $C$ intersects its image a finite number of times. (Figure 13.)

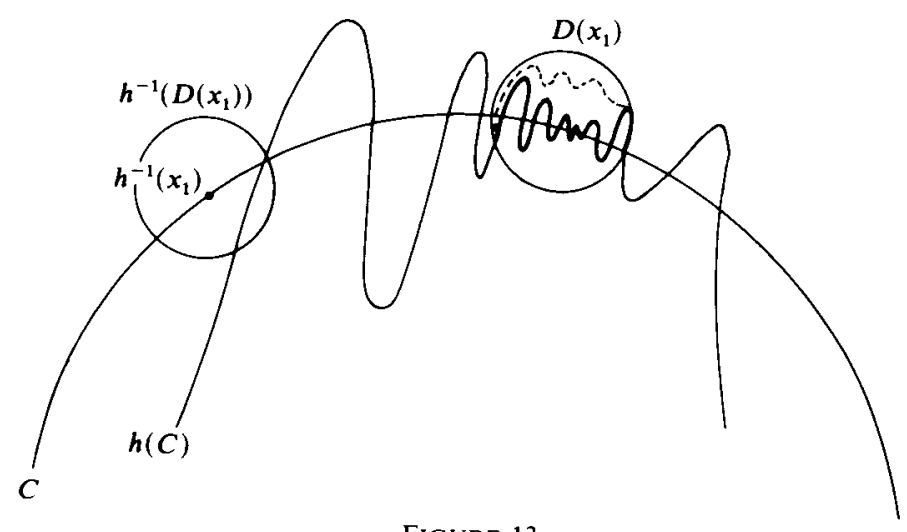

FIGURE 13 
Finally, consider the case where $D \cap h(D)$ is not connected. By the above, we can assume that $D \cap h(D)$ is the union of a finite number of components $K_{i}$, $i=1,2, \ldots, n$, with $p_{1}$ in $K_{1}$. We will construct a simple closed curve $C_{1}$ bounding a disc $D_{1} \subset D$ with $p_{1}$ in $D_{1}$ and a modified homeomorphism $h^{\prime}$ so that $h^{\prime}\left(D_{1}\right) \cap D_{1}$ has fewer components. (See figure 14.)

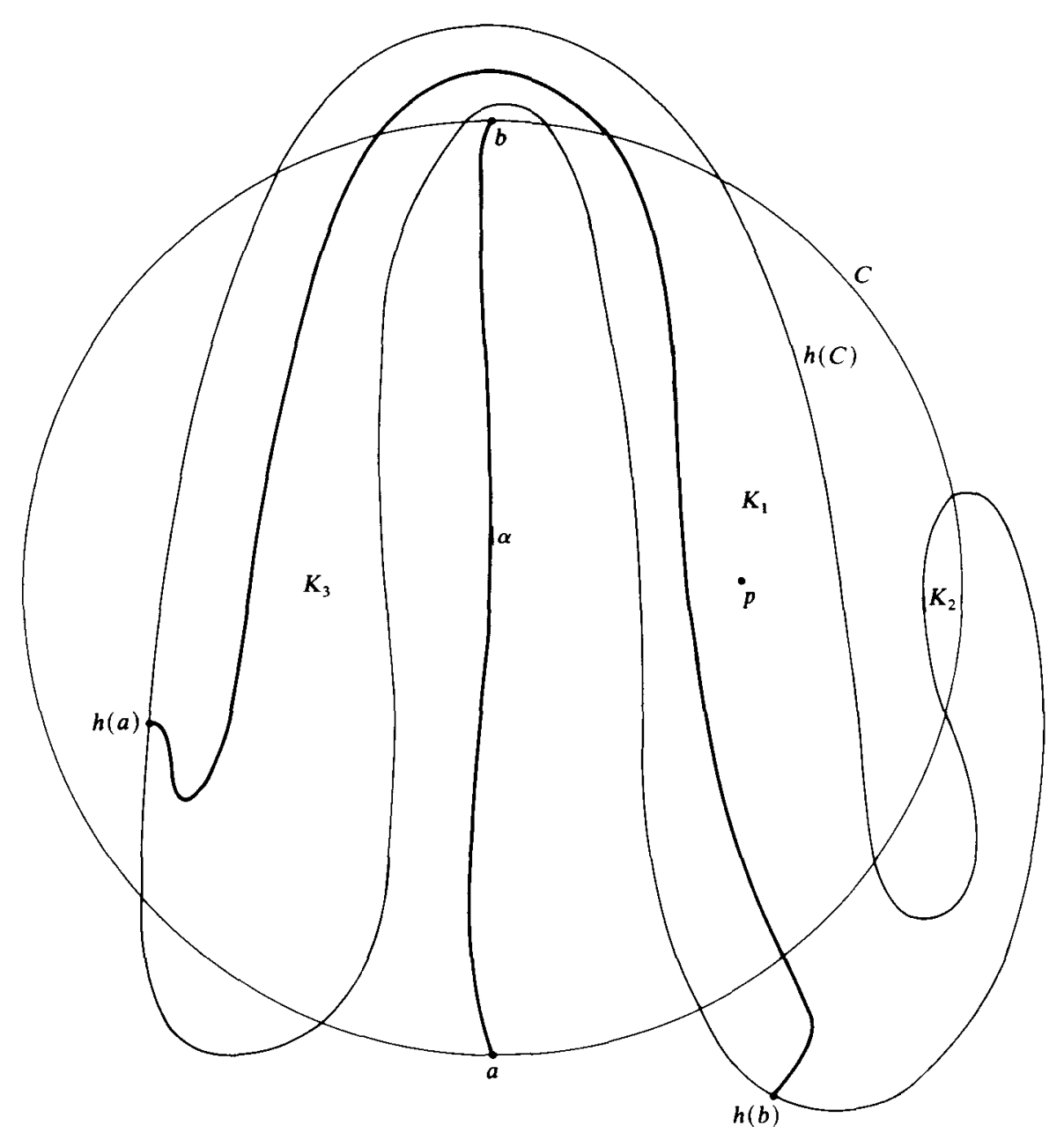

FIGURE 14

Pick $a, b \in D \cap\left(\bigcup K_{i}\right)^{c}$ such that $a, b$ are endpoints of an arc $\alpha$ in $D \cap\left(\bigcup K_{i}\right)^{c}$ and so that $\alpha \cap$ bd $\left(D \cap h(D)^{c}\right)=\{a, b\}$ and such that $\alpha$ separates $K_{1}$ from at least one $K_{i}$ in $D$. Since $h(\alpha) \cap \alpha=\varnothing$, we may assume that $h(\alpha)$ intersects $C$ a finite number of times. (Otherwise, a free modification of $h$ on a disc containing $\alpha$ will produce this condition.) 
Let $\beta_{1}$ and $\beta_{2} \subset C$ be the two arcs with endpoints $a$ and $b$. If $h(\alpha) \cap K_{i}$ is connected for each $i$, let $D_{1}$ be the disc bounded by $\alpha$ and either $\beta_{1}$ or $\beta_{2}$ - whichever is such that $K_{1} \subset D_{1}$. Then $D_{1} \cap h\left(D_{1}\right)$ has fewer than $n$ components.

Thus we assume that $h(\alpha) \cap K_{i}$ is not connected for at least some $i$. (See figure 15.) Let $x_{1}, x_{2}, \ldots, x_{m}$ be the intersection points of $h(\alpha)$ with $C$. Assume that the

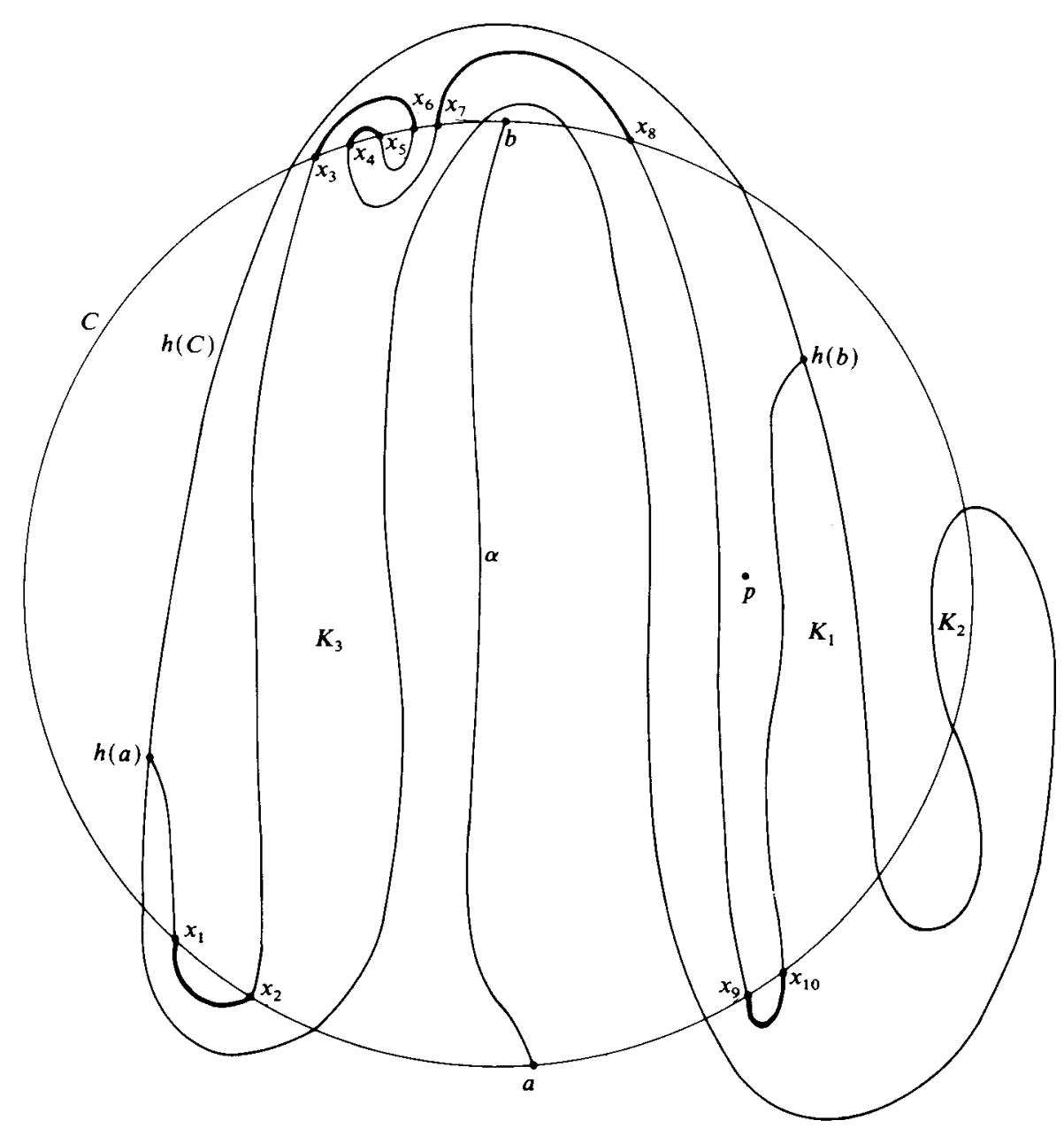

FIGURE 15

subscripts give an order to these points which is inherited from $\alpha$. Let $x_{i} x_{i+1} \subset h(\alpha)$ be the arc with endpoints $x_{i}$ and $x_{i+1}$.

Consider only those arcs which lie in the complement of the interior of $D$. Since $h(D)$ is contractible there exists at least one such arc $x_{j} x_{j+1}$ with endpoints lying in bd $\left(K_{i}\right)$ for some $i$. 
Pick $x_{j}$ so that no other $x_{k}$ lies between $x_{j}$ and $x_{j+1}$ on $C \cap$ bd $\left(K_{i}\right)$, where $x_{k} x_{k+1}$ is another such arc. We will modify the homeomorphism $h$ in such a way as to move the arc $x_{j} x_{j+1}$ into the interior of $D$. Since we will focus upon this particular arc, we will rename the arc $x y$, and the component $K$. (See figure 16.)

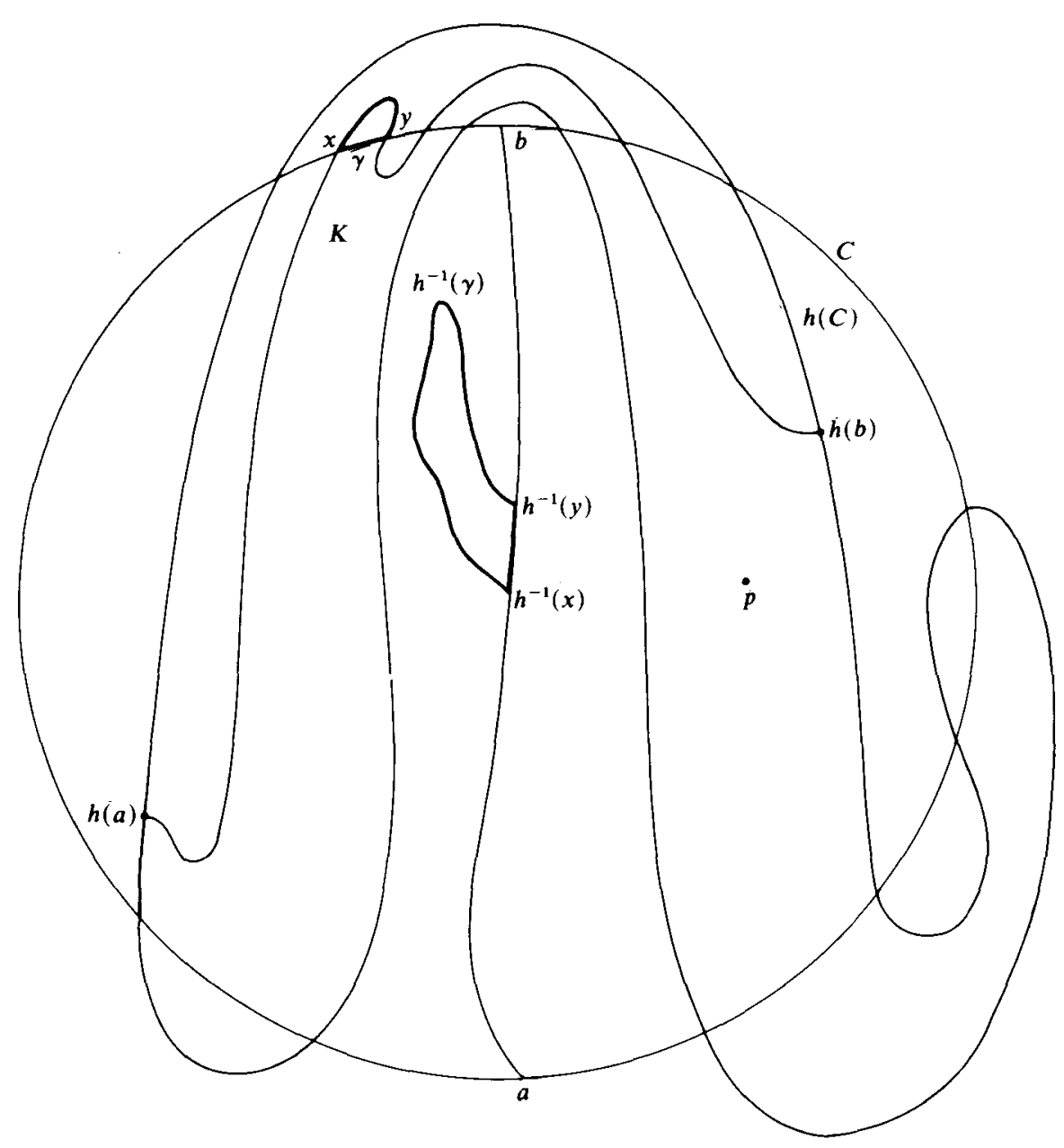

FIGURE 16

Let $\gamma$ be the arc on $C \cap$ bd $(K)$ with endpoints $x$ and $y$. We observe that $(\gamma \cup x y) \cap h^{-1}(\gamma \cup x y) \neq \varnothing$. Hence, by using a disc $E$ containing $h^{-1}(\gamma \cup x y)$ in its interior, we can modify $h$ to $h^{\prime}$ where $\left.h^{\prime}\left(h^{-1} x y\right)\right) \subset$ int $(D)$. We have pushed the arc $x y$ into the interior of $D$. Thus we can modify the map $h$ to obtain a map $h^{\prime}$ so that $h^{\prime}(\alpha) \cap K_{i}$ is connected for all $i$. 


\section{REFERENCES}

[1] K. Boucher. Private communication.

[2] M. Brown. A new proof of Brouwer's Lemma on translation arcs. Houston J. Math. 10 (1984), 35-41.

[3] M. Brown. Private communication.

[4] M. Brown. Homeomorphisms of two-dimensional manifolds. To appear in Houston J. Math.

[5] B. Schmitt. Sur les plongements, admettant zero ou un point fixe, du disque dans le plan. Topology 14 (1975), 357-365.

[6] C. Simon. A bound for the fixed-point index of an area-preserving map with applications to mechanics. Inventiones Math. 26 (1974), 187-200. 